\title{
Assessment of local muscle fatigue by NIRS - development and evaluation of muscle suit -
}

\author{
Yoshiki Muramatsu ${ }^{*}$ and Hiroshi Kobayashi
}

\begin{abstract}
Although the machinery automation technology and robotics have made significant progress in making production and distribution easy and efficient, there is still a need for manual work such as nursing and other manufacturing work. Those work could possibly cause the work-related disorders. In order to reduce the risk of work-related injury or illness, we have been developing a compact and lightweight wearable robot "Muscle Suit" to provide direct and physical motion supports for assisting human motion. The development of the Muscle Suit becomes possible through the use of the McKibben artificial muscle. This type of artificial muscle has the advantages of being compact, lightweight and reliable. The wearable Muscle Suit has high versatility and conveyance, it supports the user physically with the capability of loading more weights. The evaluation of effectiveness is a key point in the development of the Muscle Suit and it could be estimated by evaluating the muscle fatigue. The evaluation of muscle fatigue is required not only for muscle suit but also for rehabilitation, muscle training and human physical support. Although the frequency transition of electromyogram (EMG) method is generally used for fatigue assessment, it has certain drawbacks depending on conditions and environment. For instance, it is impossible to apply it in the case of isotonic contraction. There are no apparatus or methods which measure muscle fatigue noninvasively and simply. In this study, we used near-infrared spectroscopy (NIRS) to measure the serum oxygenated hemoglobin and deoxygenated hemoglobin concentration of wearer's muscle indirectly, the new term $\Delta \mathrm{Hbt}$ has been defined and the degree of fatigue has also been evaluated. And then, we had the evaluation experiment of muscle suit using blood oxygenation and the new term $\Delta \mathrm{Hbt}$ to estimate the effects of muscle suit.
\end{abstract}

Keywords: Muscle suit; Fatigue estimation; Muscle fatigue; Haptic sensor; Near-infrared spectroscopy

\section{Background}

The European Agency for Safety and Health at Work reports that Musculoskeletal Diseases account for 30\% to $46 \%$ of all work-related sick leave [1]. Based on USA's National Institute for Occupational Safety and Health, $67 \%$ of nursing care personnel and $84 \%$ of automobile factory workers have back pain as work-related disorders [2]. For workers, there are income losses when they are out of work due to work-related disorders. Furthermore, for organizations and factories, the industrial accident compensation is also an unexpected rise in production costs., Thus, we come to a conclusion that work-related disorder is a major and/or serious societal issue.

We therefore have been developing the wearable muscle suit [3-9] directly support the motion of upper

\footnotetext{
* Correspondence: yoshi@kobalab.com

Department of Mechanical Engineering, Tokyo University of Science, Tokyo, Japan
}

body as shown in Figure 1. The purpose is to provide daily assistance and help users to move unaided. It could also be useful in rehabilitation and aiding manual workers. Use of the McKibben artificial muscle makes muscle suits lightweight and practical. The motivation to develop the muscle suits was originally to support the physically challenged sufferers, although practical use is still difficult with ethical and safety issues. We then decided to apply them to manual workers for solving the problem of work-related disorders. The technology of muscle suits is fairly mature and more than 1000 visitors at the related exhibition have tried it on so far. We then investigate the market research and have been developing the production design.

In the process of development, the evaluation method of muscle suit becomes as important as the development itself. Evaluation of muscle fatigue is required not only for muscle suit but also for rehabilitation, muscle 


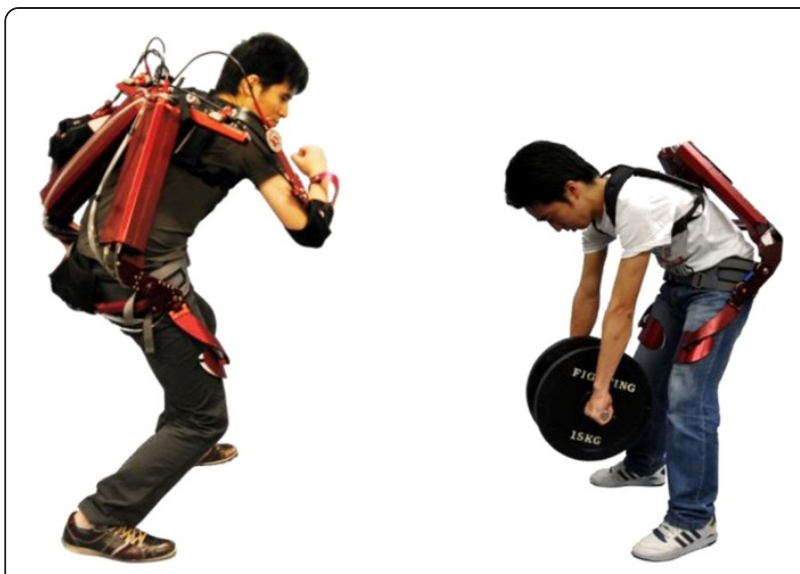

Figure 1 Muscle suit (arm + back support, back support).

training and human physical support, estimation of muscle fatigue is required. In order to prevent the economic losses such as the compensation of the workrelated injuries, the fatigue evaluation for workers is also desired. Although the frequency transition of electromyogram (EMG) method is generally used for fatigue assessment, it has certain drawbacks depending on conditions and environment. For instances, it is impossible to apply in the case of isotonic contraction. Thus, there are no apparatus or methods which measure muscle fatigue noninvasively and simply [10].

We therefore propose a method of muscle fatigue assessment using near-infrared spectroscopy (NIRS) [11]. NIRS is a noninvasive optical measurement method of blood oxygenation based on changes in the near-infrared absorption characteristics of hemoglobin $(\mathrm{Hb})$ in vivo due to the state of oxygen bonding. In general, NIRS is used for visualization of brain activity, and several studies have been done on the measurement of blood oxygenation by NIRS [12-15], which indicates the possibility of assessing muscle fatigue using NIRS. However, there is no research so far explaining the relation between blood oxygenation and muscle fatigue directly.

In this study, values of oxygenated and deoxygenated hemoglobin obtained by NIRS are employed for muscle fatigue estimation. Under the specific operation, we acquire these values and investigate the relation between muscle fatigue and those values. Then we define the index which seems to represent muscle fatigue using values of oxygenated and deoxygenated hemoglobin. In the paper written by us [16], we discussed the comparison of $\Delta \mathrm{Hbt}$ of non-state fatigue and exhaustion state in the biceps brachii, and in the other paper [17], we discussed the comparison of $\Delta \mathrm{Hbt}$ of exhaustion state of low load and exhaustion state of high load in the biceps brachii. And then using the defined index, we have an experiment of the muscle suit, and we discuss the comparison of the change in blood oxygenation with and without muscle suit in the erector spinae.

In this paper, muscle suit is introduced in chapter 2, NIRS is explained in chapter 3, index for indicating muscle fatigue is described in chapter 4 , muscle fatigue assessment and the experiment of muscle suit is discussed in chapter 5 .

\section{Configuration and performance of muscle suit McKibben artifical muscle}

The McKibben artificial muscle was selected as the muscle suit actuator because of its light weight, simple structure, softness, waterproof finish and power-toweight ratio [18]. The McKibben actuator was developed in the 1950s and 1960s in artificial-limb research [19]. The McKibben artificial muscle consists an internal bladder surrounded by a braided mesh shell with flexible, non-expandable threads attached at either end. As shown in Figure 2, when the internal bladder is pressurized, air pushes against inner surfaces and the external shell, hence it gets expanding. Due to the non-expandability of threads in the braided mesh shell, the actuator is shortened as its volume increases and/or produces a load if it is coupled to a mechanical load. This results in 35\% contraction without load and over $5 \%$ for a $150 \mathrm{~kg}$ load in case of 1.5 inch in diameter $\left(D_{0}\right)$.

\section{System configuration}

The McKibben actuator requires a compressor, a microprocessor and an electric valve with a pressure sensor, as shown in Figure 3. The electric valve controls compressed air output based on the microprocessor signal. The major drawback is the compressor. However, the factory where the muscle suit is to be used provides compressed air piping, we could use it for the muscle suit by connecting tubing to piping. For use in the home,

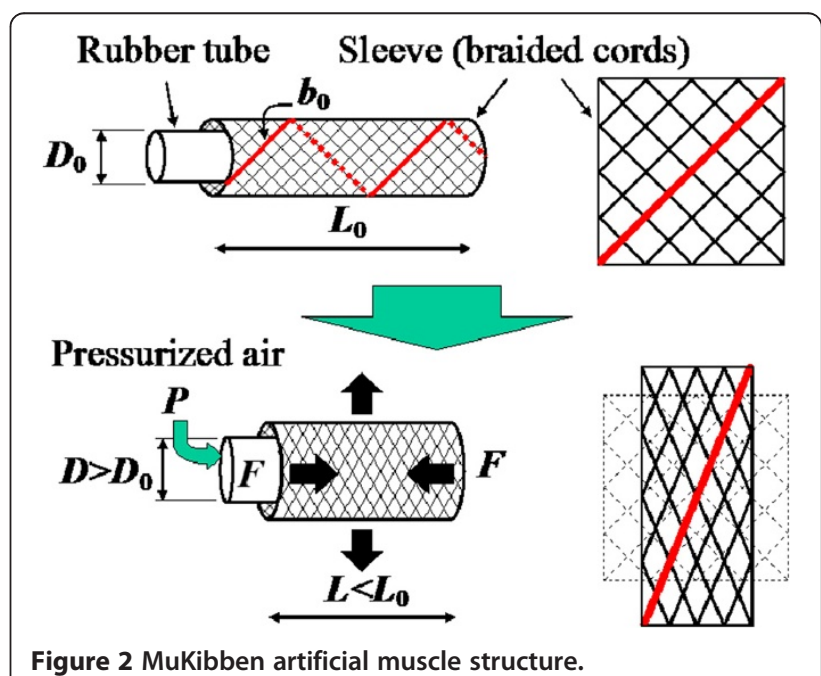




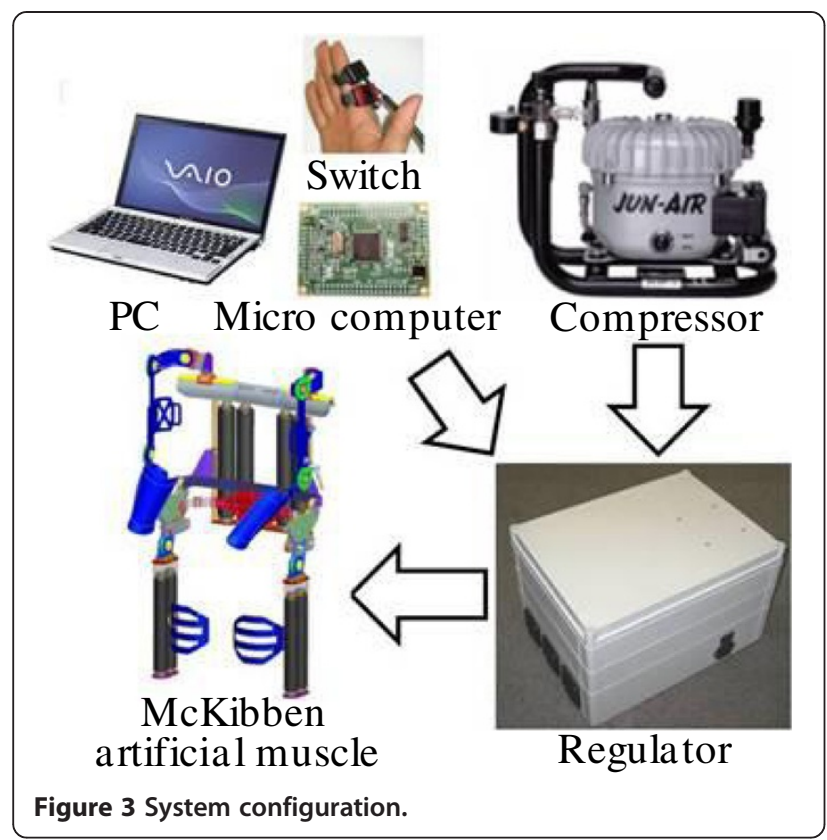

compressed air could be supplied conceivably by settingup the piping from the ceiling. As a result, the muscle suit can be made practical.

\section{Muscle suit specification and effects}

The structure of muscle suit is the exoskeleton type that does not interfere with the movement of the wearer. As shown in Figure 4, the McKibben artificial muscle and the pulley are connected with the wire, and if the McKibben artificial muscle contracts, power will be transmitted to the pulley through the wire. Pulley's diameter of each joint is $50 \mathrm{~mm}$, and one or two McKibben artificial muscles are connected to each pulley. One McKibben artificial muscle generates torque of $45 \mathrm{Nm}$.

Muscle suit schematics is shown in Figure 5. The muscle suit's mechanical structure must work smoothly with user movement. Assuming shoulder movement has 6 DOF and the center of rotation changes with arm positioning, as a result, the shoulder movement becomes complicated. Thus we developed the special structure enabling shoulder movement as illustrated [20] using 4

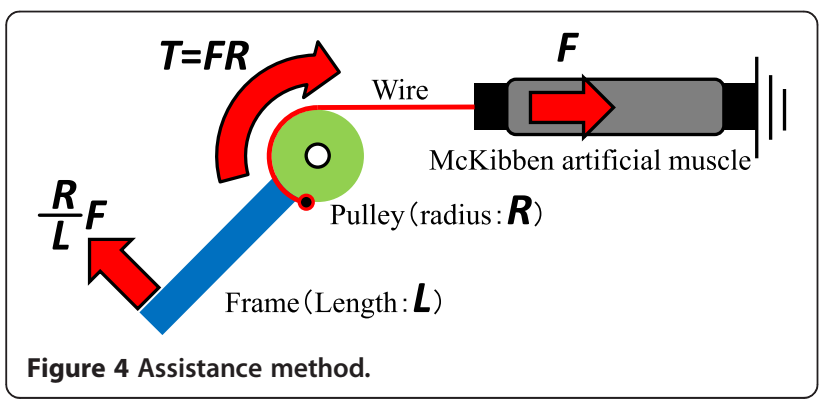

DOF for the shoulder: 3 orthogonal axes for rotation and a slider. Passive sliding follows changes in the center of shoulder rotation. The support torque of shoulders and elbows are $45 \mathrm{Nm}$, and the waist is $90 \mathrm{Nm}$.

\section{Near-infrared spectroscopy (NIRS)}

NIRS is a noninvasive method for measuring oxygenation concentration in the blood using near-infrared ray. It was commercialized in 1996 [21] by Hitachi Co. Ltd. basically for showing brain activity and has been used for brain science.

The basic principle is illustrated in Figure 6. Nearinfrared light is transmitted through the body from one probe to another. In NIRS, oxygenation concentration is a process involves hemoglobin. Hemoglobin concentration is calculated by the Lambert-Beer law, i.e.,

$$
-\log \left(I_{\text {out }} / I_{\text {in }}\right)=\varepsilon \times C \times D
$$

where $I_{i n}$ is the light input to the target muscle, $I_{\text {out }}$ is the transmitted light pass through the body, $D[\mathrm{~mm}]$ is the transit distance, $\varepsilon[1 /(\mathrm{mM} \cdot \mathrm{mm})]$ is the extinction coefficientand $C[\mathrm{mM}](\mathrm{M}: \mathrm{mol})$ indicates concentration we would like to have.

Because in real use, the influence of disturbance, such as physiological motion and the existence of outside light, the Lambert-Beer law is modified for applying the light-scattering medium as shown below

$$
-\log \left(\Delta I_{\text {out }} / I_{\text {in }}\right)=\varepsilon \times \Delta C \times D+\Delta S
$$

where $\Delta I_{\text {out }}$ is the change in transmitted light pass through the body, $\Delta C$ the change in concentration, $D$ the average optical path lengthand $\Delta S$ the change in the scattering effect. Equation (2) is called the Modified Lambert-Beer law.

In general, oxygenated hemoglobin gives oxygen to each part of the body including the muscles and then changes into deoxygenated hemoglobin. The deoxygenated hemoglobin obtains oxygen from the lungs and then changes into oxygenated hemoglobin. Note that the total amount of oxygenated hemoglobin + deoxygenated hemoglobin in the whole body should be the constant because the blood flow is constant. If the muscle is used, the oxygen is consumed there, and more oxygen is demanded. As a result, oxygenated hemoglobin is provided to the oxygen-consumed area, the ratio of oxygenated hemoglobin increases and deoxygenated hemoglobin decreases. Thus we may say that by observing oxygenated hemoglobin and deoxygenated one, muscle fatigue can be estimated.

In this study, we employed the Spectratech OEG-16 (Spectratech, Inc.) [22] in order to measure oxygenated 


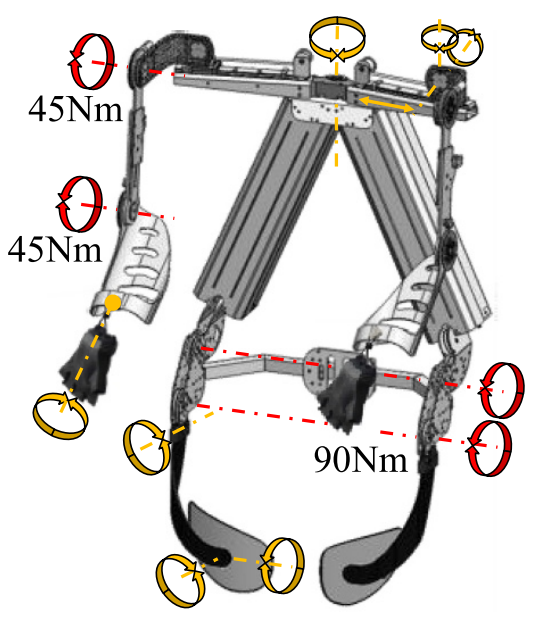

(a) Armes \& back support

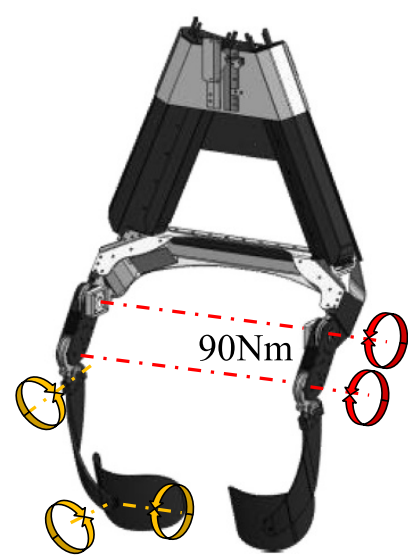

(b) Back support

Figure 5 Suit schematics.

and deoxygenated hemoglobin. As mentioned before, two values, i.e., oxygenated hemoglobin $(\Delta C$ Coxy) and deoxygenated one ( $\Delta C$ doxy), are necessary to the measurement. Also because $D$ is difficult to acquire, $\Delta$ Coxy $\cdot D$ and $\Delta C$ doxy $\cdot D$ are calculated using equations below.

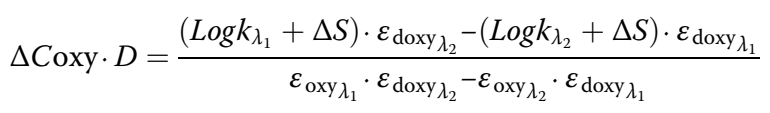

$$
\Delta C \text { doxy } \cdot D=\frac{\left(\log k_{\lambda_{1}}+\Delta S\right) \cdot \varepsilon_{\operatorname{oxy}_{\lambda_{2}}}-\left(\log _{\lambda_{2}}+\Delta S\right) \cdot \varepsilon_{\operatorname{oxy}_{\lambda_{1}}}}{\varepsilon_{\operatorname{oxy}_{\lambda_{2}}} \cdot \varepsilon_{\operatorname{doxy} \lambda_{1}}-\varepsilon_{\text {oxy } \lambda_{1}} \cdot \varepsilon_{\operatorname{doxy} \lambda_{2}}}
$$

$$
k_{\lambda}=\Delta I_{\text {out } \lambda} / I_{\text {in } \lambda}
$$

where $I_{\text {in } \lambda}$ denotes the incident light (wavelength $\lambda$ ) to the body, $\Delta I_{\text {out } \lambda}$ the change in transmitted light pass through the body, $\varepsilon_{\text {oxy } \lambda}$ and $\varepsilon_{\text {doxy } \lambda}$ the extinction coefficient of oxyhemoglobin and deoxyhemoglobin. Note that the Spectratech OEG-16 employs $770 \mathrm{~nm}$ and $840 \mathrm{~nm}$ for $\lambda_{1}$ and $\lambda_{2}$ which corresponds to $805 \mathrm{~nm}$ (where $\varepsilon_{\text {oxy }}$ and $\varepsilon_{\text {doxy }}$ inverts as shown in Figure 7) $\pm 35 \mathrm{~nm}$ selected empirically.

\section{Methods}

Index to estimate muscle fatigue by NIRS

By analyzing the relation between $\Delta C$ oxy $\cdot D$ and $\Delta C$ doxy . $D$, we propose the index which seems to represent the muscle fatigue. Following experiments are given approval of International University of Health and Welfare.

\section{Effect of various conditions}

There are individual differences exist between subjects, such as gender, age, physique, affects blood oxygenation [23]. The straining during operation is called as the
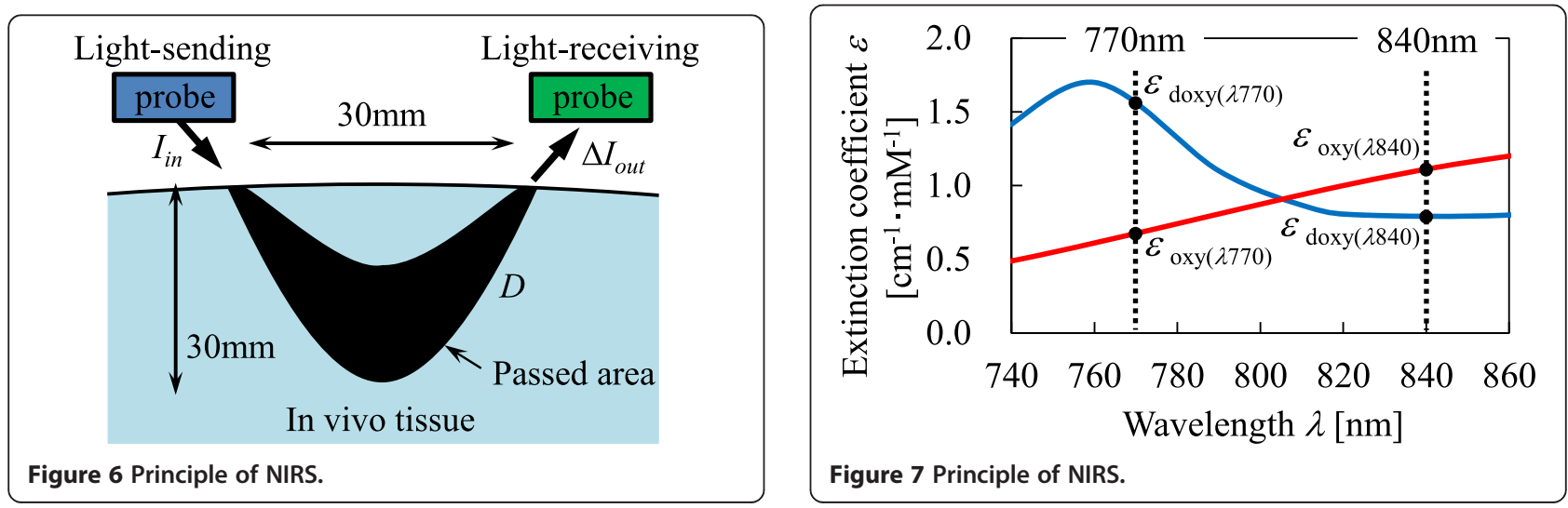


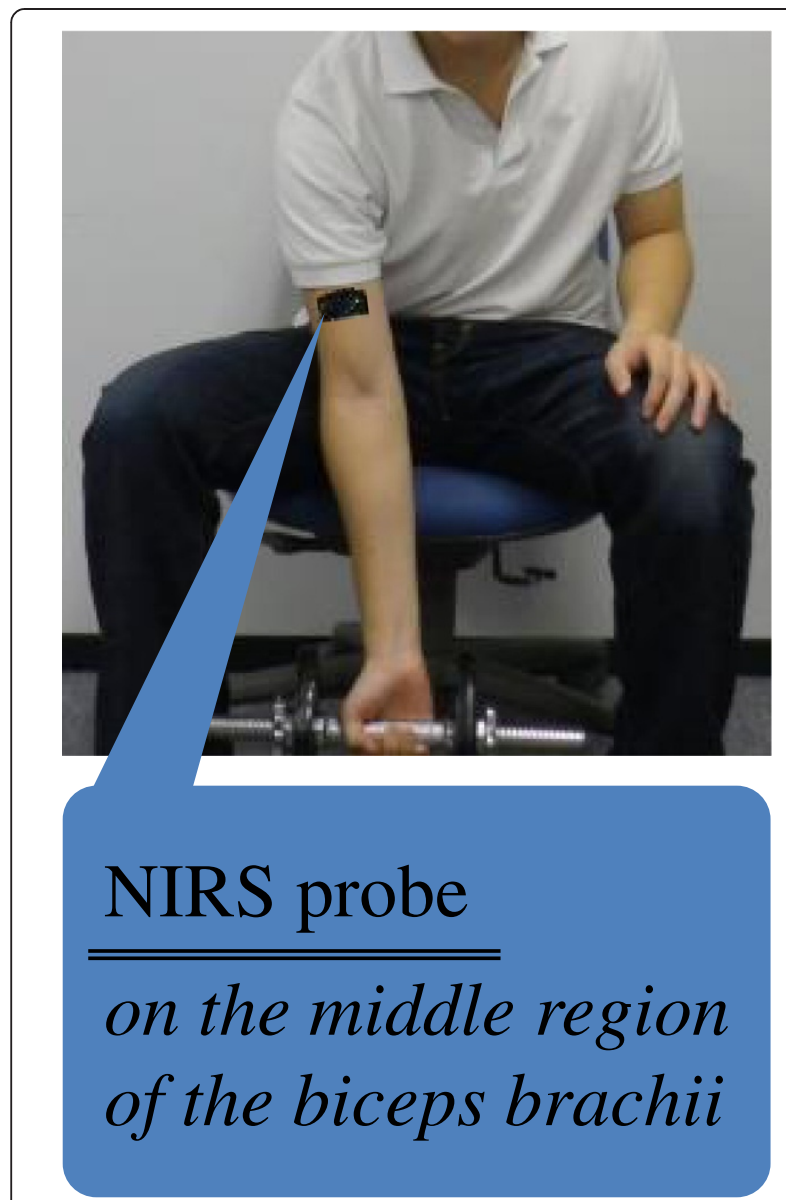

Figure 8 Measurement sites.

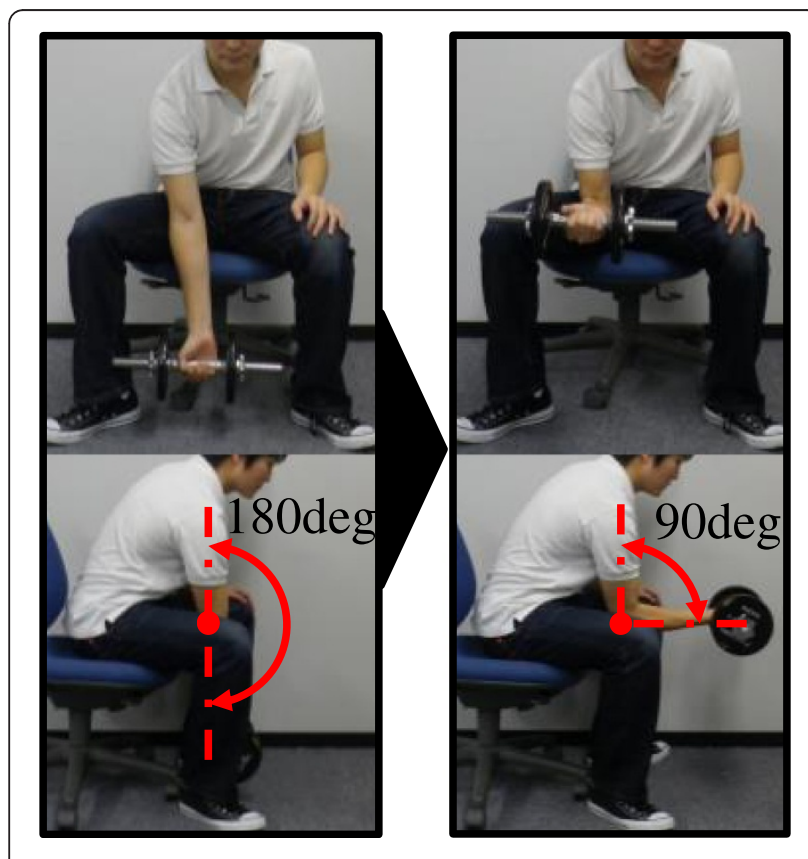

Figure 9 Evaluation experiments.

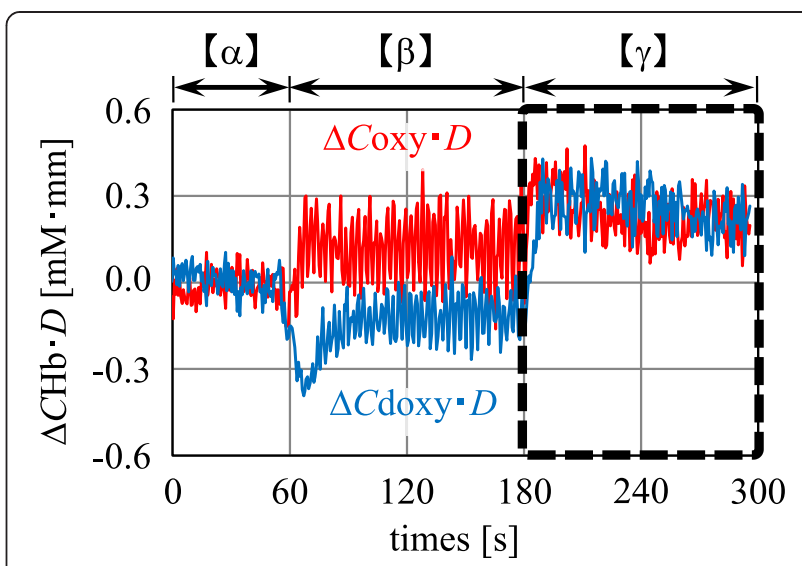

Figure 10 The result of experiment - Load of $2.5 \mathrm{~kg} \times 40$ times (sub. X).

Valsalva maneuver and physical exercise before the operation also influences blood oxygenation [24].

We then employ subjects who are the same gender, age, physique, and also ask them to rest 10 minutes before the operation and not to strain during the operation in order to avoid uncertainly factors for blood oxygenation.

\section{Preliminary experiment}

As shown in Figure 8, NIRS probe was attached in the middle area of the biceps brachii. As shown in Figure 9, we request the subject to sit on a chair, hold the dumbbell with the arm extended downward, and then lifts the dumbbell by flexing the elbow to 90 degrees. The experiment consists 3 terms, i.e., 60 seconds rest without holding any object $(\alpha), 40$ times dumbbell lifting (every 3 seconds. 120 seconds in total) $(\beta)$, and then 120 seconds rest without holding any object $(\gamma)$. We apply $2.5 \mathrm{~kg}$ and $5.0 \mathrm{~kg}$ weights. Four male adults in their 20s (X, Y, V, W) are employed for the experiment.

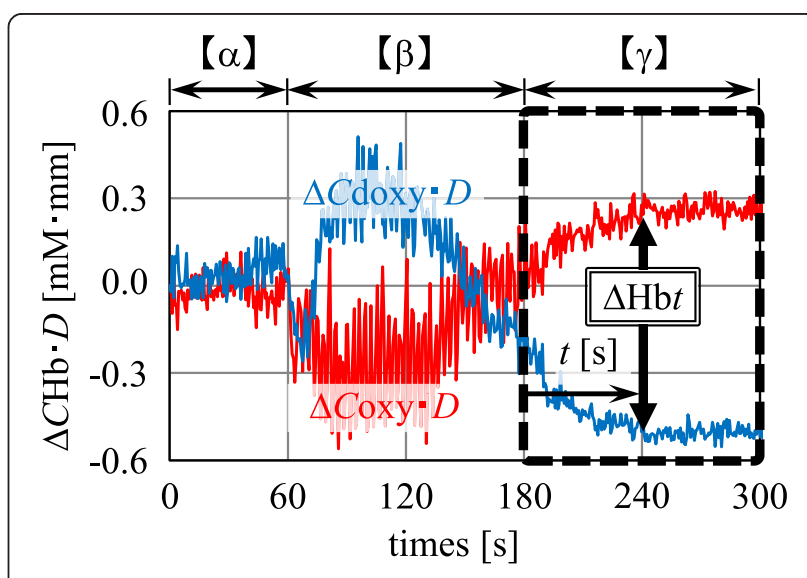

Figure 11 The result of experiment - Load of $5.0 \mathrm{~kg} \times 40$ times (sub. X). 


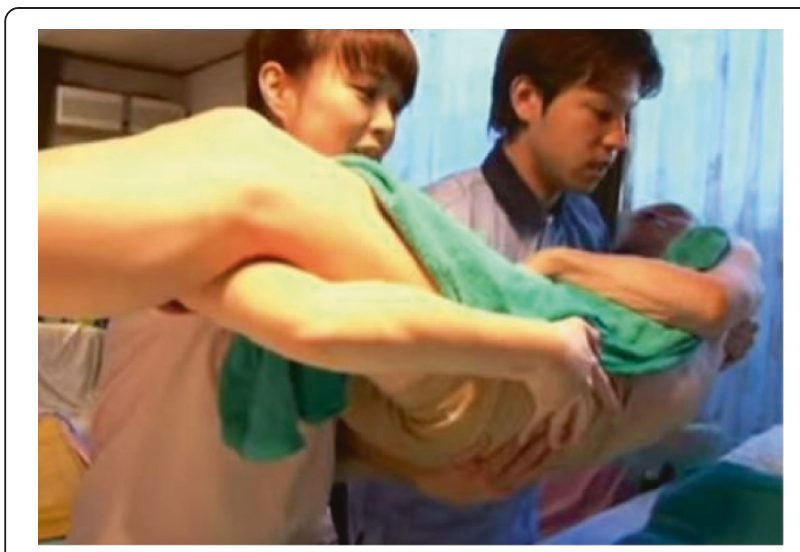

Figure 12 Home-visit bathing service. Source: Asahi sun clean. Co., Ltd.

\section{Definition of $\Delta \mathrm{Hbt}$}

Figure 10 and Figure 11 show the results obtained by subject $\mathrm{X}$ for $2.5 \mathrm{~kg}$ and $5.0 \mathrm{~kg}$ weights. We find that each term, i.e., pre-operation: rest ( $\alpha ; 60$ seconds), operation ( $\beta ; 120$ seconds), and post-operation: rest $(\gamma ; 120$ seconds) are clearly identified. There are no differences at term $\alpha$ between $2.5 \mathrm{~kg}$ and $5.0 \mathrm{~kg}$ weights. In contrast to term $\alpha$, there is no similarity in terms of subjects and weights at term $\beta$. Whereas when we focus on the difference between $\Delta C$ oxy $\cdot D$ and $\Delta C$ doxy $\cdot D$, clear feature is observed, i.e., Figure 11 is bigger than Figure 10 with respect to the difference between $\Delta C \operatorname{coxy} \cdot D$ and $\Delta C \operatorname{doxy} \cdot D$. A similar trend is observed for all other subjects as well.

We then define $\Delta \mathrm{Hbt}$ shown in equation (6) as an index for representing muscle fatigue where $t$ indicated the elapsed time after term $\beta$ as expressed in Figure 11.

$$
\Delta \mathrm{Hb} t=\Delta \operatorname{Coxy} \cdot D-\Delta C \text { doxy } \cdot D
$$

\section{Results and discussion}

Review and evaluation of muscle suit in actual operation by NIRS

In this section, we verify the effects of muscle suit in the scenario of home visit bathing service using back support muscle suit. In home-visit bathing service, the hardest physical work for nursing staff is to carry patients from bed to bath and vice versa as shown in Figure 12.

\section{Experimental set up}

Therefore, we measure the blood oxygenation of erector spinae muscles of nursing staff while they carrying patients from bed to bath as Figure 13 indicates.

The operation of moving patients is performed by two people in cooperation. One is responsible for carrying the lower side of body and the other is in charge of the upper side. The upper side of body accounts for $70 \%$ of whole body weight, as a result, the subject in charge of the upper body uses the muscle suit. In this experiment, the height of bed is set to $750 \mathrm{~mm}$, which is an average value of beds using in the actual home-visit bathing service. The distance between bed and bath is set to $900 \mathrm{~mm}$. Furthermore, the height of bath is set to $500 \mathrm{~mm}$ as the bath used is the standard type of everyday bathing service. The weight of the user of bathing service is set to $60 \mathrm{~kg}$. Subjects perform the operation carrying the patient from bed to bath, up and down, in 12.5 seconds, similarly, from bath to bed in 12.5 seconds as well. The total 25 seconds operation is considered as one cycle, subjects perform 5 cycles of operation in a row as the prior investigation suggests after 5 times the fatigue reaches the peak value. We then compare blood oxygenation in case of using muscle suit and not using muscle suit.

Two home-visit bathing service staffs (A: 25 years of age, $175 \mathrm{~cm}$ height, $75 \mathrm{~kg}$ weight, B: 43 years of age, $180 \mathrm{~cm}$ height, $80 \mathrm{~kg}$ weight) are employed. As Figure 14 illustrates, the NIRS probes are attached to the left and right side of erector spinae muscles.

\section{The results of an experiment}

First, Figure 15 shows the result of subject A's right side erector spinae of 5 th cycle's $\Delta \operatorname{Coxy} \cdot D$ with muscle suit. Figure 15 shows changes of $\Delta \operatorname{Coxy} \cdot D$ during the time of operation. From these results, we can conclude that the changes of $\Delta \operatorname{Coxy}^{\prime} D$ fluctuate greatly over the period of time. With the knowledge of this, we then check the results of blood oxygenation of subject A and B.

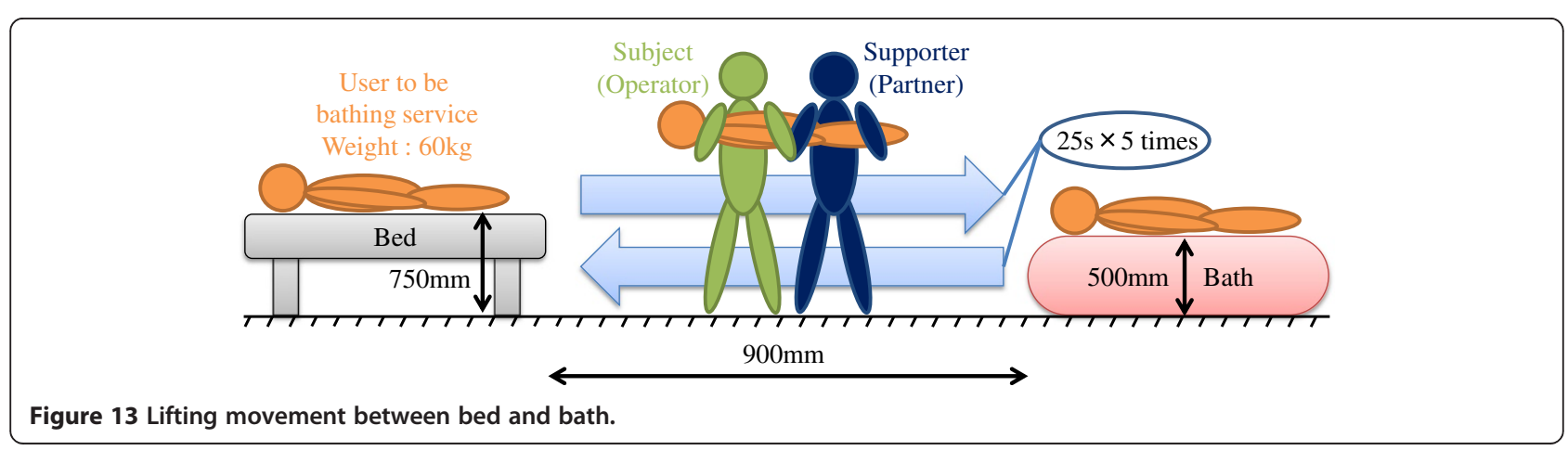




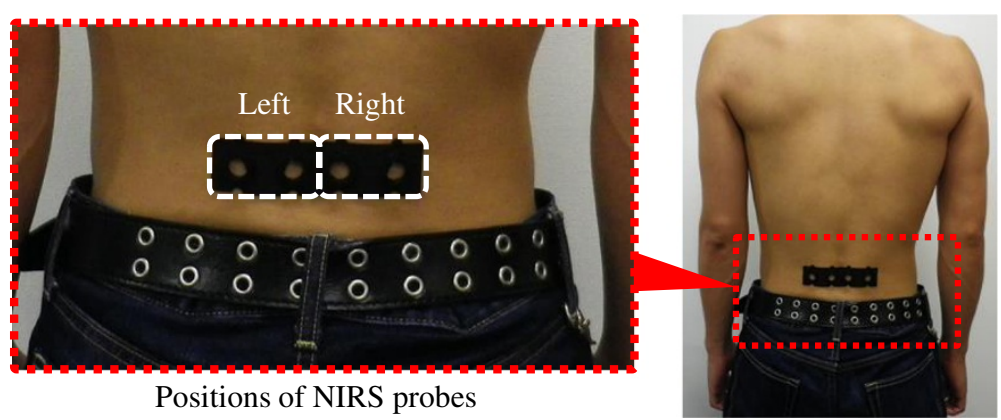

Figure 14 Measurement sites.

Figures 16, 17, 18, 19 show the time shift of blood oxygenation, $\Delta C \operatorname{Coxy} \cdot D$ and $\Delta C \operatorname{doxy} \cdot D$, of each cycle. Figure 16 and Figure 17 show the results of subject $A$. From Figure 16a and Figure 17a, there is almost no difference in the change of $\Delta C o x y \cdot D$ with or without muscle suit. However, from Figure 16b and Figure 17b, the result of $\Delta C$ doxy $D$ without muscle suit is largely positive compared with the result with muscle suit. In particular, the difference is significant in carrying state in Figure 16b. Figure 18 and Figure 19 show the results of subject B. From Figure 18a and Figure 19a the result of $\triangle C \operatorname{oxy} \cdot D$ in case of without muscle suit during interval is largely negative compared with the result with muscle suit. And from Figure 18b and Figure 19b, there is almost no difference in the change of $\Delta C$ doxy $D$ with or without muscle suit.

In conclusion, the change in blood oxygenation with or without muscle suit may vary between subjects, but it is clear that there is a difference in blood oxygenation due to the occupying of muscle suit. Particularly, to analyze the results by using $\Delta \mathrm{Hb} t$ shown in Figure 20 and Figure 21, we can see that the trend of $\Delta \mathrm{Hb} t$ during carrying operation between bed and bath without muscle suit is large negatively compared with the result with muscle suit. In addition, with the knowledge that Figure 20a is a special case, we can see that the trend of $\Delta \mathrm{Hb} t$ with muscle suit is changing gradually compared with the result without muscle suit. Here, as for the result of Figure 20a, comparing the results of $\Delta C \operatorname{Coxy} \cdot D$ in Figure 16 and in Figures $17,18,19$, we can see that the fluctuation of $\Delta C$ oxy $\cdot D$ in Figure $16 \mathrm{a} 2$ is large. It is clear that there is a difference between the change in Figure $16 \mathrm{a} 2$ and in Figures 17, 18, 19. The reason for this may be the different ways of occupying muscles when subject doing the operation.

\section{Conclusion}

We have proposed a new method for evaluating local muscle fatigue using NIRS. We focus on the change in oxygenated and deoxygenated hemoglobin under the operation and define $\Delta \mathrm{Hbt}$ as the differences between them. In the experiment, as for the muscle of biceps brachii, we find that $\Delta \mathrm{Hbt}$ is constant in case of nonfatigue. Whereas in case of exhaustion, it increases according to elapsed time and shifts upward following with cycles.

We conducted an investigation of blood oxygenation during carrying operation between bed and bath in home-visit bathing service. It can therefore be concluded that there is the difference in blood oxygenation by occupying muscle suit or not. In addition, not only the measurement of blood oxygenation after operation but also during the operation could possibly be evaluated in real time to assess the muscle fatigue. The evaluation
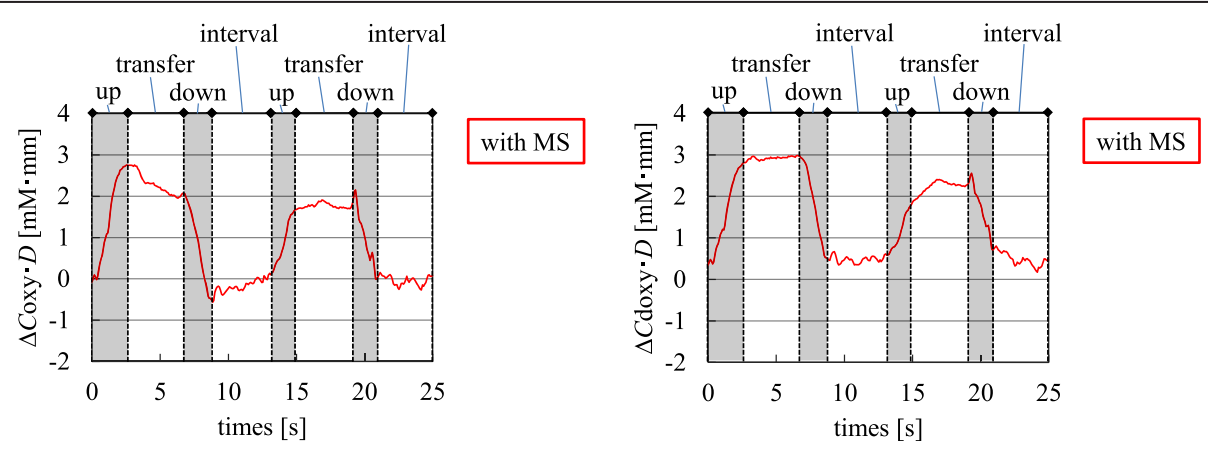

Figure 15 The result of experiment - subject A's right side erector spinae muscles of 5th cycles (with muscle suit (MS)) -- 


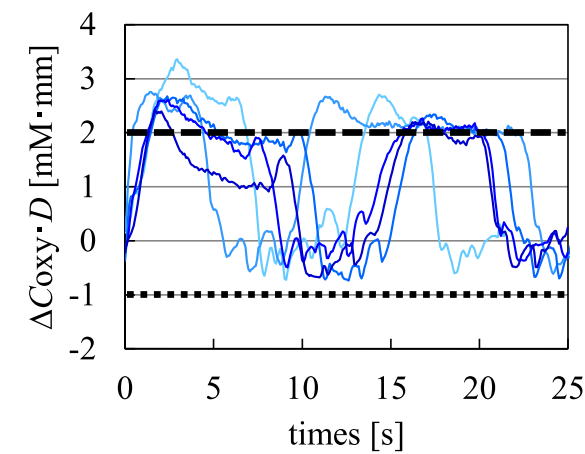

(a1) Resule of $\Delta C$ oxy $\cdot D$ without MS

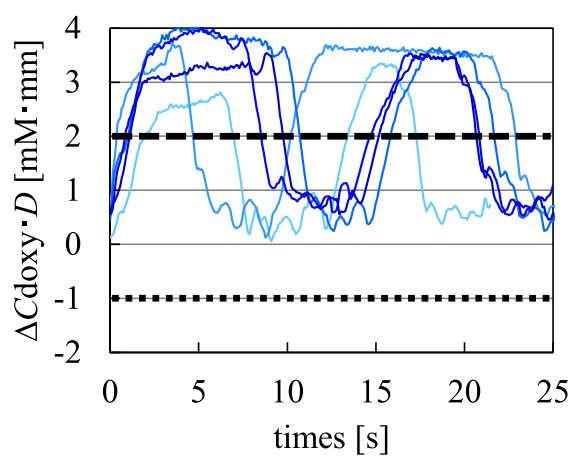

(b1) Resule of $\Delta C$ doxy $\cdot D$ without MS
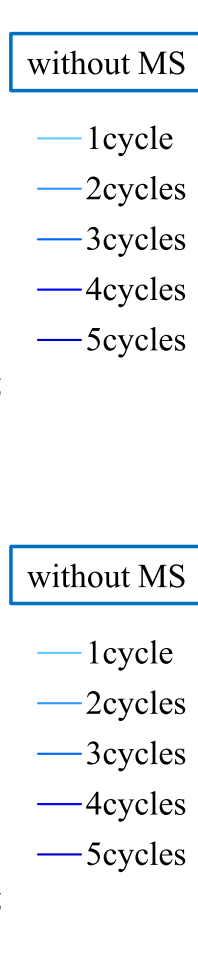
- 1 cycle
- 2cycles
-3cycles
-4cycles
-5 cycles

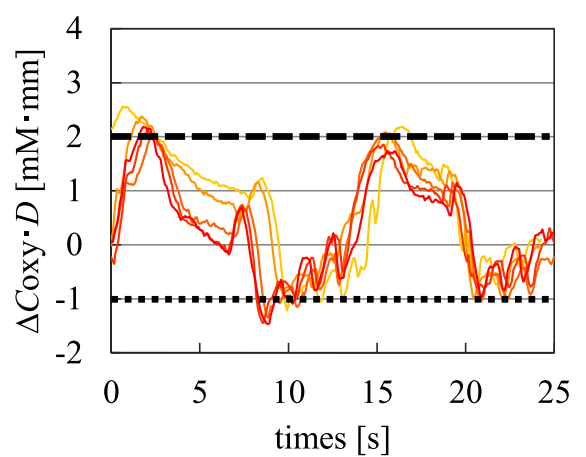

(a2) Result of $\Delta C$ oxy $\cdot D$ with MS

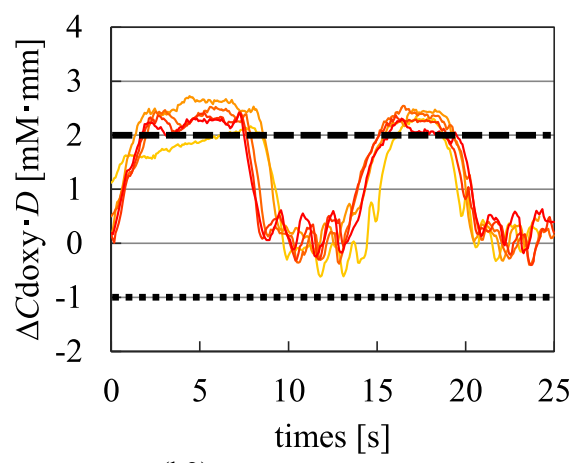

(b2) Result of $\Delta C$ doxy $\cdot D$ with MS with MS

- 1cycle

-2cycles

-3cycles

-4cycles

-5 cycles

Figure 16 The result of experiment - Erector spinae muscles of left - (sub. A).

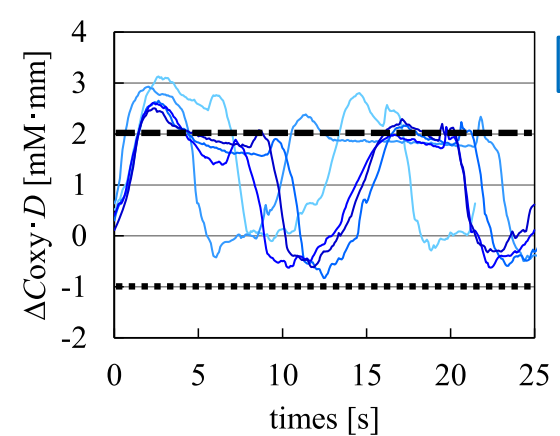

(a1) Resule of $\triangle C \operatorname{Coxy} \cdot D$ without MS

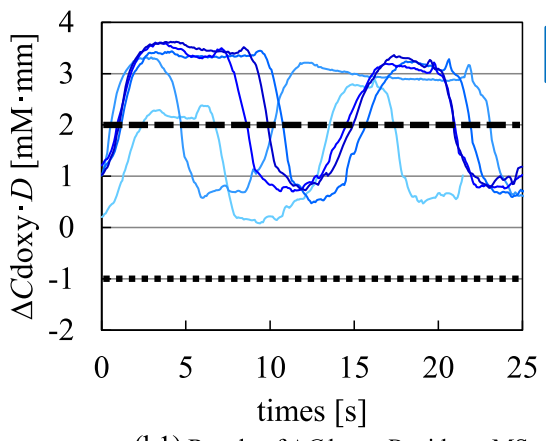

(b1) Resule of $\Delta C$ doxy $\cdot D$ without MS
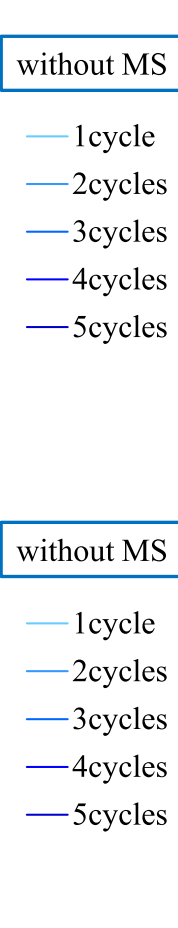

Figure 17 The result of experiment - Erector spinae muscles of right - (sub. A).

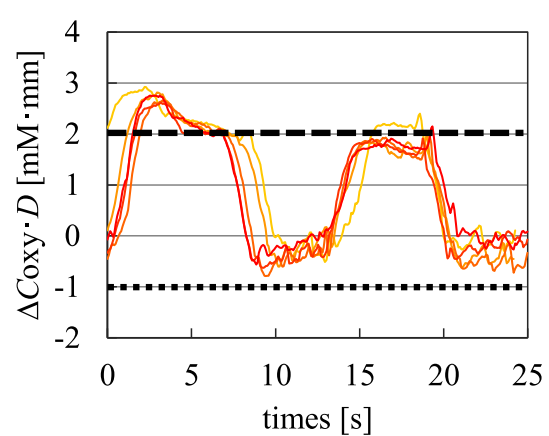

(a2) Result of $\Delta C$ oxy $\cdot D$ with MS

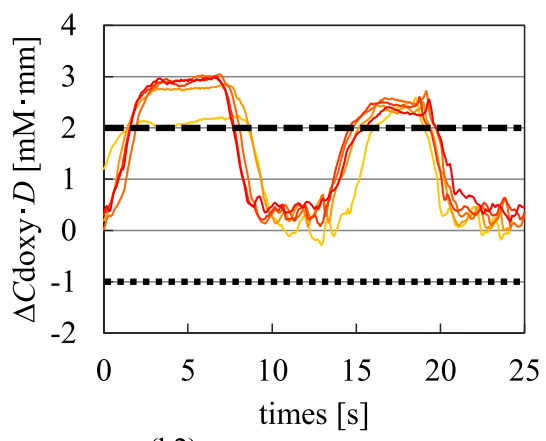

with MS

- 1cycle

-2cycles

-3cycles

-4cycles

-5 cycles 


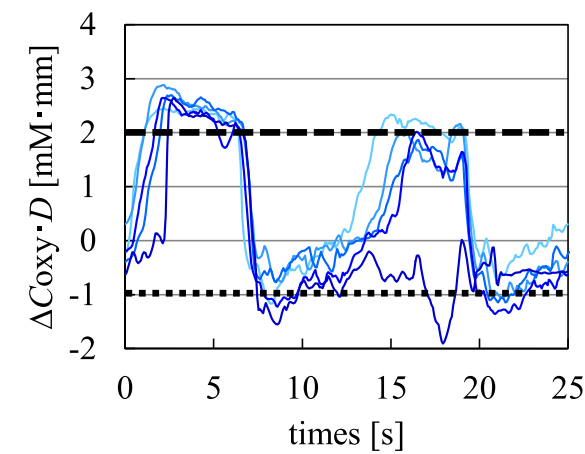

(a1) Resule of $\Delta C$ oxy $\cdot D$ without MS

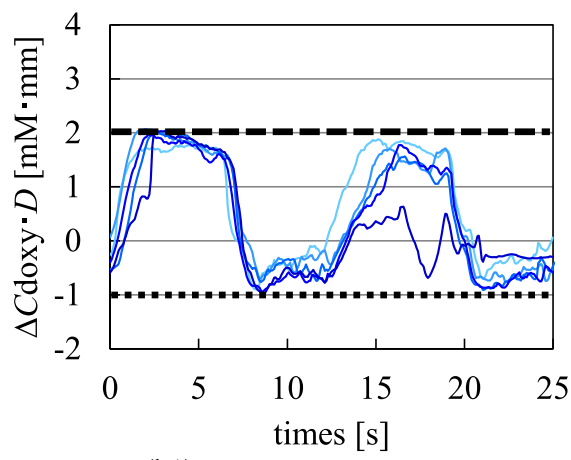

(b1) Resule of $\Delta C$ doxy $\cdot D$ without MS
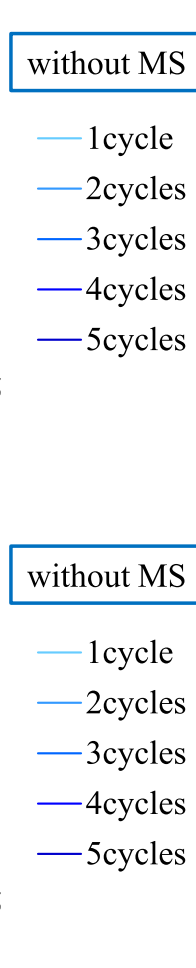

Figure 18 The result of experiment - Erector spinae muscles of left - (sub. B).

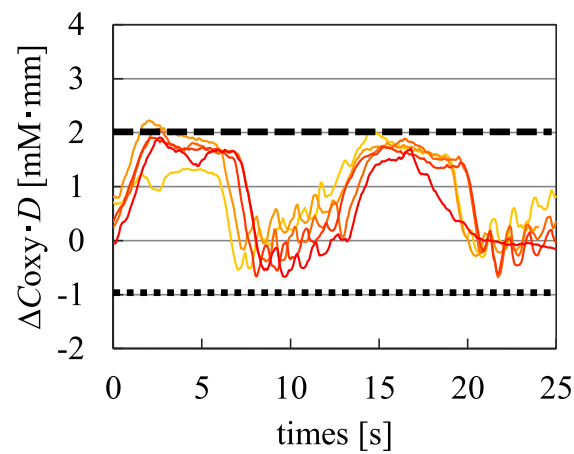

(a2) Result of $\Delta C$ oxy $\cdot D$ with MS

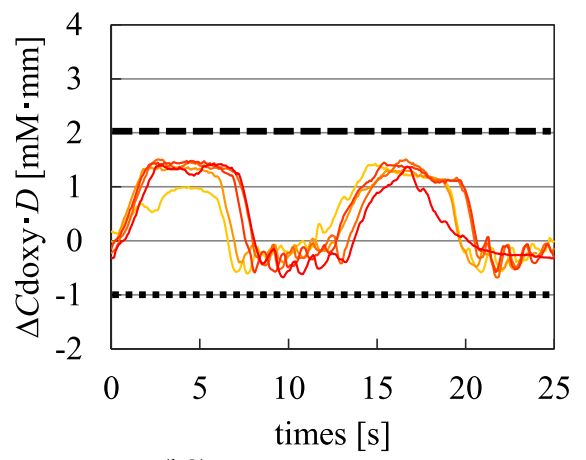

with MS

- 1 cycle

-2cycles

-3cycles

-4 cycles

-5 cycles

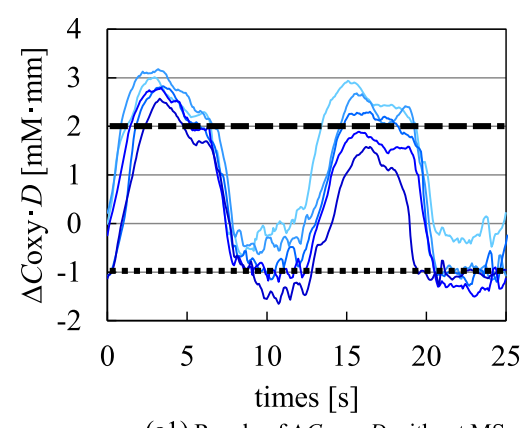

(a1) Resule of $\Delta C$ oxy $\cdot D$ without MS

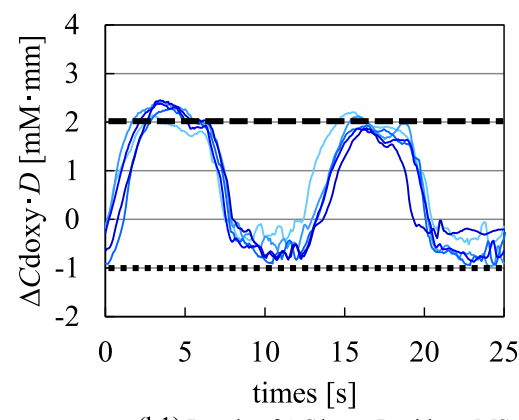

(b1) Resule of $\Delta C$ doxy $\cdot D$ without MS

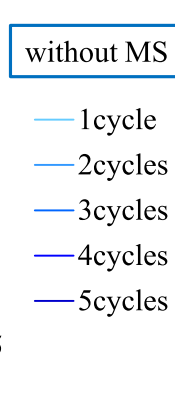

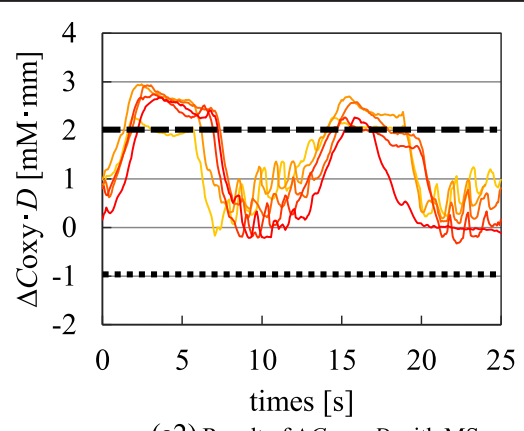

(a2) Result of $\Delta C$ oxy $\cdot D$ with MS

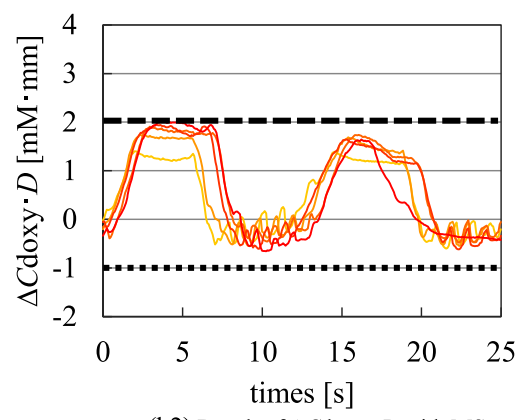

(b2) Result of $\Delta C$ doxy $\cdot D$ with MS

\section{with MS \\ - 1 cycle \\ - 2cycles \\ - 3cycles \\ - 4cycles \\ -5 cycles}

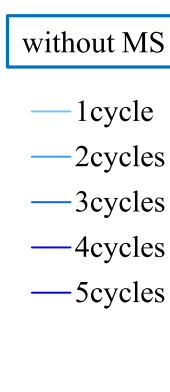

muscles of right - (sub. B). 


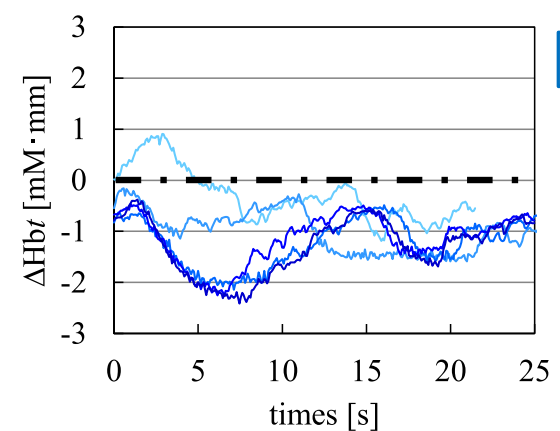

(a1) Resule of $\Delta \mathrm{Hb} t$ of left side without MS
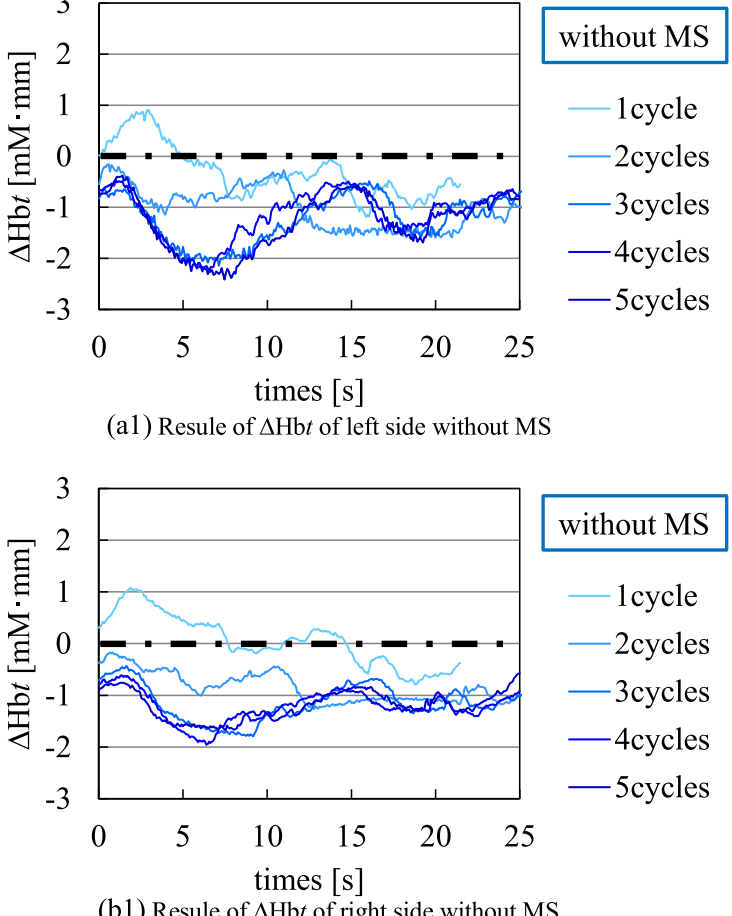

Figure $\mathbf{2 0}$ The result of experiment - Erector spinae muscles - (sub. A).

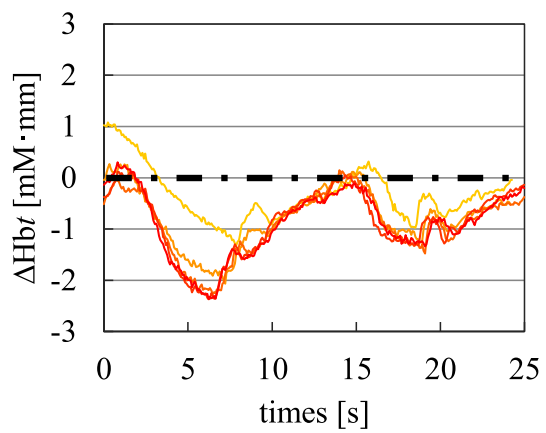

with MS

-1 cycle

-2cycles

- 3cycles

-4cycles

-5 cycles

(a2) Result of $\Delta \mathrm{Hb} t$ of left side with MS

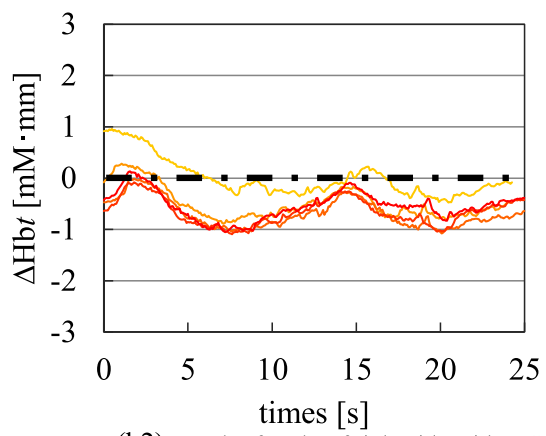

with MS

-1cycle

-2cycles

-3cycles

- 4cycles

-5 cycles

(b2) Result of $\Delta \mathrm{Hb} t$ of right side with MS 
method of NIRS could be used in further research of assessing the effects of using muscle suit.

Investigation of local muscle fatigue assessments by NIRS will continue, and adaptation to various scenes require assessment of muscle fatigue such as medical treatment, welfare, the work place and evaluation of muscle suit will be considered.

\section{Consent}

Written informed consent was obtained from the patient for the publication of this report and any accompanying images.

\section{Competing interests}

The authors declare that they have no competing interests.

\section{Authors' contribution}

Prof. HK is responsible for research of the muscle suit. YM carried out experiments in this paper and drafted the manuscript. Both authors read and approved the final manuscript.

Received: 15 January 2014 Accepted: 27 October 2014

Published online: 18 November 2014

\section{References}

1. European Agency for Safety and Health at Work. http://osha.europaeu/en/ publications/factsheets/9. Accessed 6 Feb 2014

2. National Institute for Occupational Safety and Health. http://www.cdc.gov/ niosh/docs/97-141/ergotxt3.html. Accessed 14 Jan 2014

3. Kobayashi H, Matsushita D, Ishida Y, Kikuchi K (2002) New robot technology concept applicable to human physical support -the concept and possibility of the muscle suit (wearable muscular support apparatus) -. J Robot Mechatr 14(1):46-53

4. Kobayashi H, Shiiban T, Ishida Y (2004) Realization of All 7 motions for the upper limb by a muscle suit. J Robot Mechatr 16(5):504-512

5. Kobayashi H and Suzuki H (2005) Development of a New Shoulder Mechanism for a Muscle Suit. Paper presented at IEEE/RSJ International Conference on Intelligent Robots \& Systems (IROS'05) Edmonton, Alberta, Canada, 2-6 Aug 2005, TAll-13:pp.2727-2732

6. Hiroshi K, Hirokazu N (2007) Development of Muscle Suit for Supporting Manual Worker. IEEE/RSJ International Conference on Robot and Systems Sanya, China, 29 Oct - 2 Nov 2007, pp.1769-1774

7. Muramatsu $Y$, Kobayashi $H$, Sato $Y$, Jiaou H, Hashimoto $T$ and Kobayashi $H$ (2011) Quantitative Performance Analysis of Muscle Suit - Estimation by Oxyhemoglobin and Deoxyhemoglobin. In: Proceedings of 2011 IEEE International Conference on Robotics and Biomimetics, Phuket, 7-11 Dec 2011, pp 293-298

8. Muramatsu Y, Kobayashi H, Sato Y, Jiaou H, Hashimoto T, Kobayashi H (2011) Quantitative performance analysis of exoskeleton augmenting devices -muscle suit- for manual worker. Int J Autom Technol 5(4):559-567

9. Muramatsu $Y$, Umehara $H$, and Kobayashi $H$ (2013) Improvement and Quantitative Performance Estimation of the Back Support Muscle Suit. Proceedings of The 35th International Conference on the IEEE Engineering Medicine and Biology Society (EMBC 2013), Osaka, 3-7 July 2013, pp 2844-2849

10. Japan Industrial Safety and Health Association. http://www.jisha.or.jp. Accessed 14 Jan 2014

11. Maki A, Obata A (2009) Development and outlook from near-infrared spectroscopy to optical topography. J Jpn Coll Angiol 49(2):121-130

12. Oka T (1999) Measurement of oxidative metabolism in the isometric exercise of medial vastus muscle by near infrared spectroscopy: a study on diagnostic indices and their reliabilities. Jpn J Rehabil Med 36(8):526-532

13. Yamamoto K (2006) Measurement of dynamic change in muscle oxygenation using near-infrared spectroscopy. J Jpn Soc Stomatognath Funct 12:93-99

14. Motohashi K, Kiryu T, and Chigira T (2006) On-Site Evaluation of Muscle Fatigue During Repetitive Exercise. IEICE Technical Report MBE2006-11, IEICE, Tokyo, pp.41-44
15. Yoneyama S, Kudo N, and Yamamoto K (2004) Simulation of muscle metabolism for analyses of muscle oxygenation measure by NIRS-Steady and transient responses to exercise-. IEICE Technical Report MBE2004-12, IEICE, Tokyo, pp.5-8

16. Muramatsu $Y$ and Kobayashi $H$ (2012) Muscle Fatigue Assessment using NIRS. Proceedings of the 1st Annual IEEE Healthcare Innovation Conference of the IEEE EMBS, Houston, USA, 7-9 Nov 2012, pp 223-226

17. Muramatsu Y and Kobayashi H (2013) Assessment of Local Muscle Fatigue by NIRS. Proceedings of the 2013 7th International Conference on Sensing Technology ICST 2013 Wellington, New Zealand, 3-5 Dec 2013, pp 624-630

18. Chou CP, Hannaford B (1996) Measurement and modelling of McKibben pneumatic artificial muscles. IEEE Trans Robot Autom 12:90-102

19. Schulte HF Jr (1961) The characteristics of the McKibben artificial muscle. In: The Application of external power in prosthetics and orthotics. National Academy of Sciences-National Research Council, Washington D. C.

20. Kobayashi H, Aida T, Hashimoto T (2009) Muscle suit development and factory application. Int J Autom Technol 3(6):709-715

21. Hitachi Medical Corporation. http://www.hitachi-medical.co.jp/product/opt/ index.html. Accessed 6 Feb 2014

22. Spectratech Inc. http://www.spectratech.co.jp/En/indexEn.html. Accessed 6 Feb 2014

23. Isao K, Nobuhito I, K Kazumi, Masato S, Hidekatsu T (2003) Differences in muscle oxygenation level according to gender during isometric hand-grip exercises. J Hokkaido Univ Ed 54(1):89-96

24. Hanya S (2010) Effects of the valsalva maneuver on the mechanical properties of the main pulmonary artery: relationship to pulse wave reflection. J Jpn Coll Angiol 50(2):189-195

doi:10.1186/s40648-014-0019-2

Cite this article as: Muramatsu and Kobayashi: Assessment of local muscle fatigue by NIRS - development and evaluation of muscle suit -. $R O B O M E C H$ Journal 2014 1:19

\section{Submit your manuscript to a SpringerOpen ${ }^{\odot}$ journal and benefit from:}

- Convenient online submission

- Rigorous peer review

- Immediate publication on acceptance

- Open access: articles freely available online

- High visibility within the field

- Retaining the copyright to your article

Submit your next manuscript at $>$ springeropen.com 